\title{
Proceso de enseñanza aprendizaje de la escritura a partir de la lectura de la realidad
}

\author{
Teaching process learning writing from the reading of reality
}

\section{Processo de ensino aprendendo a escrever a partir da leitura da realidade}

\author{
ARTÍCULO DE INVESTIGACIÓN
}

\author{
Moisés Rubén Tinta Aruquipa \\ moisesruben.2@gmail.com
}

ORCID: 0000-0002-6276-0684

Universidad Autónoma del Beni “José Ballivian”, Bolivia

Recibido 02 de diciembre 2020 | Arbitrado y aceptado 11 de diciembre 2020 | Publicado en diciembre 2020

\section{RESUMEN}

La escritura es un elemento principal en la educación primaria, por ende la investigación tuvo el objetivo analizar el desarrollo de la escritura a partir de la lectura de la realidad en los estudiantes del primer año de escolaridad del nivel primario. La investigación fue desarrollada en el marco del método holístico, holográfico y la dialéctica. Los resultados en el campo de acción, utilizando métodos teóricos y empíricos, los resultados hecha el análisis a las pruebas pedagógicas, el $79 \%$ de presentan deficiencias en el rendimiento de la escritura, y los entrevistados ratificando que existe deficiencias en el desarrollo de la escritura, en conclusión: el desarrollo de la escritura es un proceso permanente de motivación en el desarrollo de capacidades y habilidades de percepción de los cinco sentidos, para la escritura a partir de la realidad.

Palabras clave: Enseñanza y aprendizaje; capacidades, habilidades, escritura, lectura de la realidad

\begin{abstract}
Writing is a main element in primary education, therefore the research aimed to analyze the development of writing from the reading of reality in students in the first year of primary school. The research was developed within the framework of the holistic, holographic and dialectical method. The results in the field of action, using theoretical and empirical methods, the results made from the analysis of the pedagogical tests, $79 \%$ of them present deficiencies in the performance of writing, and the interviewees ratifying that there are deficiencies in the development of writing In conclusion: the development of writing is a permanent process of motivation in the development of capacities and abilities of perception of the five senses, for writing from reality.
\end{abstract}

Key words: Teaching and learning; abilities, skills, writing, reality reading 


\section{RESUMO}

A escrita é um elemento central do ensino fundamental, portanto a pesquisa teve como objetivo analisar o desenvolvimento da escrita a partir da leitura da realidade em alunos do primeiro ano do ensino fundamental. A investigação foi desenvolvida no quadro do método holístico, holográfico e dialético. Os resultados no campo de ação, utilizando métodos teóricos e empíricos, os resultados obtidos a partir da análise das provas pedagógicas, $79 \%$ deles apresentam deficiências no desempenho da escrita, e os entrevistados ratificam que há deficiências no desenvolvimento da escrita concluindo: o desenvolvimento da escrita é um processo permanente de motivação no desenvolvimento das capacidades e habilidades de percepção dos cinco sentidos, para escrever a partir da realidade.

Palavras-chave: Ensino e aprendizagem; habilidades, habilidades, escrita, leitura da realidade

\section{INTRODUCCIÓN}

Desde tiempos remotos la humanidad buscó las formas de representación simbólica en el progreso económico, político, cultural y social con la finalidad de mantener, y transmitir diferentes conocimientos a las futuras generaciones. En ese proceso se constituyó la simbolización con diferentes grafías y símbolos que permitieron el potenciamiento de la construcción histórica de la realidad, transformándose en significado, en la evolución de las diferentes generaciones, para la comunicación progresiva con mayor sentido a través de la escritura.

Por ello, la escritura se promovió en diferentes pueblos en proceso de evolución grafológica de representación de signos, con la finalidad de contar con conocimientos sistematizados y documentados, en ese proceso la escritura es uno de los elementos muy esperados en la construcción histórica de los acontecimientos sociales de diferentes estructuras sociales.

Por lo tanto, en el proceso de enseñanza y aprendizaje de la escritura se constituye en un elemento importante en la adquisición de capacidades cognitivas y habilidades motrices, en ese sentido la escuela ha promovido una serie de metodologías para la enseñanza y aprendizaje, así mismo revisada la teoría de diferentes autores como: Baqués (2004) sostiene que, la percepción realiza la función descodificador de la realidad, así como José Castorina cita a Lev Vygotsky, (1998a) estableciendo que el carácter social del aprendizaje pasa por la Zona de Desarrollo Próximo, y Ferreiro (2005) en su estudio establece el sistema de escritura en el desarrollo del niño, estos aportes permiten la comprensión del proceso de enseñanza y aprendizaje (PEA) de la escritura.

En el contexto escolar los estudiantes del primer año de escolaridad presenta dificultades en la escritura por diferentes factores, esto visibiliza la caracteriza en el rendimiento de la escritura, por la falta de apropiación de capacidades lingüísticas puesto que, en ese proceso los estudiantes no identifican con claridad los signos, en consecuencia no se demuestra los resultados en la expresión fonética y en la construcción de la escritura, si bien el problema de aprendizaje es dependiente de varias dificultades, vacíos y problemas, en el presente caso existe una mayor dependencia de la motivación y las carencia de estrategias de innovación curricular entoces, por la imperiosa necesidad de enfatizar el PEA en la escritura se plantea, ¿De qué manera el proceso de enseñanza y aprendizaje a partir de la lectura de la realidad contribuye en la escritura?

Por consiguiente; el objetivo es, analizar el proceso de enseñanza y aprendizaje de la escritura a partir de la lectura de la realidad en 
los estudiantes del primer año de escolaridad del nivel primario.

En ese sentido la investigación caracteriza el desarrollo de la escritura y las dificultades en el proceso de enseñanza y aprendizaje de la escritura, por consiguiente, producto del análisis y reflexión al bajo rendimiento en la escritura y las necesidades de métodos en la enseñanza de la escritura, se considera una metodología centrada en la enseñanza de la escritura a partir de la lectura de la realidad del contexto de los estudiantes, lo cual contribuye a promover el estudio de la fonética y el grafema de las palabras en el desarrollo de la escritura, con la finalidad de que, los estudiantes puedan describir, significar, caracterizar y escribir ideas para consolidar las capacidades y habilidades el texto escrito

\section{MÉTODO}

El proceso de la investigación se desarrolló tomando en cuenta el tipo de investigación cualitativa en la profundización, reflexión y argumentación del proceso de construcción de conocimientos.

En el proceso de analisis del objeto de estudio se considero la entrevista a los maestro y la la aplicación de la prueba objetiva a los estudiantes del primer año de escolaridad de primaria, la recogida de información consistió en la recopilación, identificación de ideas, categorización y significación de los componentes del tema de investigación, mediante el proceso dialéctico y holístico en el análisis de la teoría y la realidad, generada mediante un proceso de análisis y síntesis que, permitió la caracterización esencial en el manejo de la información, en un proceso sistemático y organizado resumiendo lo más importante de la información general a lo específico, por consiguiente se rescata la esencia de los componentes analizados en la apropiación de los conceptos teóricos.

\section{Prueba pedagógica}

Ahora los resultados que reflejan la aplicación de la prueba pedagógica en los estudiantes para conocer la realidad en el rendimiento de la escritura en el proceso de enseñanza y aprendizaje. Inicialmente se presenta el comportamiento de los resultados según los indicadores, en la siguiente tabla 1 :

Tabla 1. Resumen de resultados de la prueba pedagógica

\begin{tabular}{|c|c|c|c|c|}
\hline \multicolumn{2}{|r|}{ Indicadores } & $\begin{array}{l}\text { No } \\
\text { Deficientes }\end{array}$ & Deficientes & $\begin{array}{c}\% \\
\text { Deficientes }\end{array}$ \\
\hline 1 & Escribe su nombre & 9 & 6 & 40 \\
\hline 2 & Escribe palabras de forma autónoma & 3 & 12 & 80 \\
\hline 3 & Escribe nombres según las imágenes & 4 & 11 & 73 \\
\hline 4 & $\begin{array}{l}\text { Considera los fonemas en la lectura de la } \\
\text { realidad para la escritura }\end{array}$ & 0 & 15 & 100 \\
\hline 5 & Las oraciones escritas tienen coherencia & 2 & 13 & 87 \\
\hline & 5 & 15 & 57 & 79 \\
\hline
\end{tabular}

Nota: Comportamiento de los indicadores "Prueba pedagógica”. 
En la tabla se expresa el comportamiento de los indicadores, según el producto de la escritura de los estudiantes en el desarrollo de la prueba pedagógica, analizada el grado de incidencia en el problema de estudio, se constata el bajo rendimiento de la escritura de los estudiantes en el primer año de escolaridad de primaria.

\section{Resultado de la entrevista}

Los resultados de la entrevista a las maestras permitieron confrontar la precaria innovación del proceso técnico pedagógico en la enseñanza y aprendizaje de la escritura en los estudiantes, así mismo se comprendió la falta de estrategias de motivación, la existencia de las dificultades en el desarrollo de capacidades y habilidades para la escritura, así como la iniciativa propia de los estudiantes para plasmar la ideas, en el desarrollo de las grafemas y la construcción de las palabras de forma autónoma.

\section{Análisis y fundamentación}

Una vez comprendida las causas que dificultan el proceso de enseñanza $y$ aprendizaje de la escritura, se considera los componentes que permiten comprender las relaciones internas y externas en el desarrollo de la escritura, asumiendo los aspectos recurrentes de la cotidianidad de los aspectos del entorno que nos rodea, promoviendo la dinámica de percepción de los órganos sensoriales, sobre la base de Marian Baques (2004), promoviendo la realimentación mediante el proceso de entrada y salida en el desarrollo de las capacidades y habilidades.

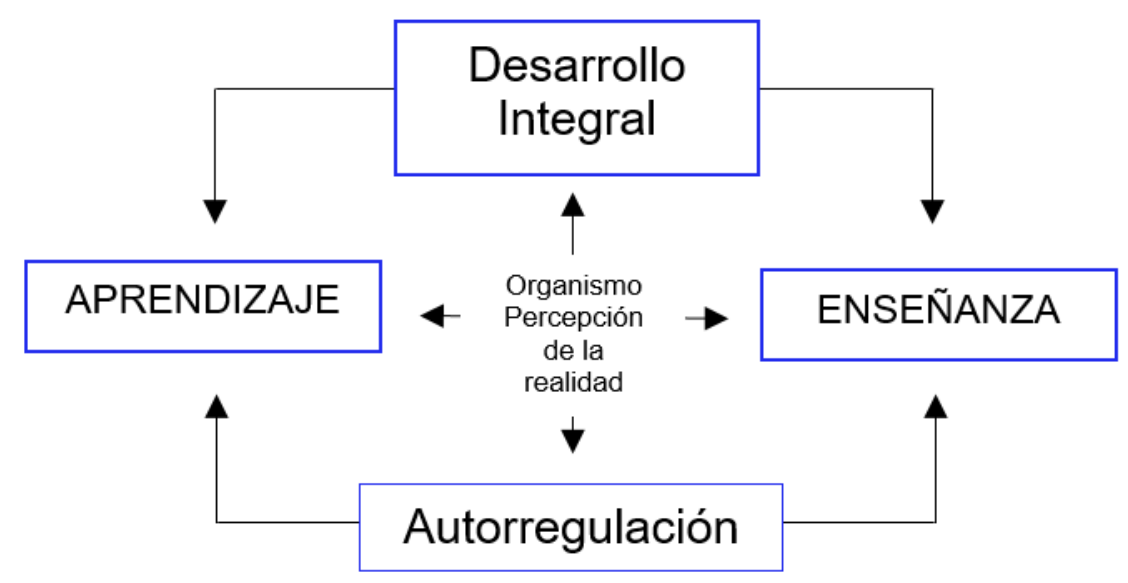

Figura 1. Esquema de procesamiento de la información.

En la figura se considera el modelo para el proceso de enseñanza y aprendizaje, considerando un proceso dinámico de autorregulación que consolida el desarrollo del organismo y la percepción de la realidad en el desarrollo integral de las capacidades y habilidades, mediante la asimilación (entrada) y la producción del aprendizaje (salida) para el desarrollo de la escritura a partir de la realidad. 


\section{Discusión}

El proceso de enseñanza y aprendizaje es eminentemente social, este proceso se constituye en una interacción e interrelación de acciones entre un grupo de individuos de dos a más personas de diversas edades, sin limitación alguna, con tal de promover la dinámica de aprendizaje y la enseñanza, sea de manera intencionada o natural en la vida escolar y cotidiana.

Para Maturana (1988) manifiesta que "el aprender tiene que ver con los cambios estructurales que ocurren en nosotros de manera contingente a la historia de nuestras interacciones" (Maturana, 1988, p. 28), por consiguiente, el aprendizaje se desarrolla de forma sistemático y dinámico, vinculado a la realidad vivencial de cada contexto, desde la realidad diversa del ambito social, cultural, economico, politico y educativo.

En ese proceso, la enseñanza se constituye en una actividad viceversa del aprendizaje, a partir de la enseñanza que brindan los contenidos de los diferentes ámbitos biopsicosociales del contexto. En ese orden de ideas, Picardo (2005) plantea que "la esencia de la enseñanza está en la transmisión de información mediante la comunicación directa o apoyada en la utilización de medios auxiliares, de mayor o menor grado de complejidad" (Picardo, 2005, p. 278), enfatizando que la enseñanza es dinámica en el conjunto de transmisiones sistemáticas de información y de conocimientos a través de la comunicación directa, en la interrelación de personas, expresadas en determinadas etapas de generación en generación. De esa manera, se recalca la importancia de la enseñanza en el aprendizaje, para promover una acción reciproca para desarrollar procesos sistemáticos de aprendizaje. Dentro de ese proceso, la enseñanza y el aprendizaje responde a las diversas actividades cotidianas en relación a los diferentes ámbitos, así como se da en el desarrollo de hábitos de higiene, en la alimentación, en el cuidado al medio ambiente, en el aprendizaje de los valores ciudadanos, estos aprendizajes se desarrollan de diferentes maneras en la dinámica social, cultural, político y educativo.

Desde la perspectiva psicopedagógica, Baqués (2004) sostiene que, "Contamos con un organismo que tiene el centro neurálgico en el cerebro, con canales de entrada y de salida" (Baques, 2004, p. 30), desataca que el proceso mental es como una caja negra que permite la asimilación de aprendizajes, en ese transcurso se desarrolla una regulación, se fortalece la enseñanza y el aprendizaje del proceso cognitivo, así como Castorina (2004) cita a Lev Vygotsky, (1998a) "La Zona de Desarrollo Próximo es la distancia entre el nivel de desarrollo real, que se suele determinar a través de la solución independiente de problemas, y el nivel de desarrollo potencial" (Castorina, 2004, pp. 24 - 25). Expresa que, el proceso cognitivo pasa por un proceso de plasticidad en el desarrollo de las neuronas, en la dinámica de la transformación permanente de la enseñanza y aprendizaje.

Dentro de este marco, Álvarez (2014) señala que, "La enseñanza es la actividad de aquel que ofrece la experiencia acumulada" y "El aprendizaje es la actividad de aquel que recibe la experiencia acumulada." (Álvarez, ob. cit., p. 13). Es por ello, que la enseñanza y el aprendizaje se hace reciproco que se da en todos los espacios necesarios de relación familiar, comunitaria y escolar, vinculada a una serie de experiencias de asimilación. Por lo tanto, el proceso de aprendizaje es relativo a la enseñanza de experiencias de maestros a estudiantes en las actividades curriculares, generando espacios graduales de interacción dedicada a promover el desarrollo de la ciencia en las diferentes áreas de saberes y 
conocimientos acudiendo a la experiencia de sus compañeros, así como la realidad del contexto aporta en el aprendizaje.

En efecto, el desarrollo de la autorregulación del organismo humano responde al proceso de percepción del medio ambiente. "Su contenido tendrá forma visual, auditiva, olfativa, dermo-cinestésica, táctil, bucal, motriz. Mantendrá el equilibrio entre el organismo y el medio en un constante feedback." (Baques, 2004, p. 18), En ese proceso la enseñanza y aprendizaje de la escritura demanda de la apropiación de capacidades y habilidades cognitivas y motrices para el desarrollo de la escritura.

Principalmente para Ferreiro (1997) "La escritura puede ser considerada como una representación del lenguaje o como un código de transcripción gráfica de la unidades sonoras" (Ferreiro, 1997, p. 13), por lo tanto, las representaciones gráficas se muestran en todo contexto comunitario y en todo momento, lo que permite entender que los niños están en permanente interacción en su vida cotidiana, en la construcción del cúmulo de conocimientos de imagen visual y auditiva para facilitar el desarrollo de la escritura. Por otra parte Amanda Rojas plantea otras influencias:

...desde el nacimiento hasta el ingreso a la escuela y en la que debe existir un sistema de influencias educativas encaminadas a garantizar el desarrollo físico, intelectual, moral y estético, o sea, el máximo desarrollo de todas las potencialidades físicas y psíquicas propias de la edad y por consecuencia se propicia una determinada preparación para la escuela (Rojas, 2000, p. 4).

De tal manera, los niños también aprenden de acuerdo a las influencias del contexto que le rodea, mediante diversas actividades de experimentación y de aprendizaje de modelos que influencian los procesos cognitivos, el desarrollo motriz, los valores y principios en la expresión escrita de conocimientos, en la expresión de las potencialidades físicas y psíquicas propias de la edad en aprestamiento de etapa inicial de los procesos de representación de mensajes de forma escrita.

En todo caso Teberosky (2000) enfatiza la apropiación de la escritura en los primeros años de la escolaridad, por lo tanto señala, "A los 5 años pueden diferenciar entre narración y otros tipos cuando se trata de rescribir textos conocidos porque tienen ideas sobre lo que es un poema, una carta, una noticia o una receta de cocina" (Teberosky, 2000, p. 9). Aunque en el proceso de aprendizaje se presentan limitaciones, por la dificultad de encarar la enseñanza adecuada según los tipos de textos y las ideas escritas en un texto, es importante destacar el sentido de los mensajes en el desarrollo de los tipos de textos.

Por ello, se considera que, la enseñanza de la escritura sea tomada en cuenta a partir de la relación de diferentes tipos de textos, en la capacidad de conocer mensajes escritos, así como las habilidades para la lectura de la realidad en el proceso de la escritura convencional de los estudiantes, desde la construcción de las primeras frases en la comprensión de aspectos complejos en el proceso de enseñanza y aprendizaje de la escritura. Por tanto, es importante considerar lo que Rojas (2000) plantea el "aprestamiento, la de adquisición de los diferentes fonemas de nuestro idioma natal, así como las necesarias habilidades caligráficas que conllevarán a que el niño se apropie de los grafemas del alfabeto que le servirán para la escritura,..." (Rojas, ob. cit., p. 2).

Esto significa que, la escritura no se desarrolla en un periodo corto de tiempo, más 
bien es importante propiciar una etapa de preparación acorde a los esquemas lógicos de desarrollo las habilidades lingüísticas, fonéticas y motrices de los niños y niñas, así mismo cabe resaltar las habilidades caligráficas es un proceso simultáneo en el desarrollo de los grafemas y morfemas en la escritura.

De esa manera se hace imprescindible el desarrollo de capacidades y habilidades para la escritura, con la preparación necesaria en el fortalecimiento de prácticas vinculadas al análisis concreto de la realidad vivencial, social, coyuntural del contexto que nos rodea, en ese sentido las acciones y la mente se encuentra en permanente interacción. Así como Rojas (2000) plantea que, "En la práctica, los niños pueden percibir, cualquier sonido que se pronuncie enfatizadamente en una palabra, por ejemplo: sssol, soool o solll. Posteriormente ellos mismos deben ser capaces de aislar cualquier sonido que se les pida en una palabra" (Rojas, ob.cit., p. 9), codificando la inportancia de la ubicación de las letras en una palabra, según la entonacion de los sonidos al momento de la lectura y la escritura.

En ese contexto los procesos de enseñanza y aprendizaje se hace importante la práctica de la percepción para desarrollar los mecanismos del sistema nervioso, estimulando los aprendizajes según presenta los signos lingüísticos de cada palabra, tanto en la construcción de la fonética y en la construcción morfológica de las palabras, es por ello que la percepción se convierte en un medio de transporte y transformación de conocimientos en el cerebro, por medio de las sensaciones, estímulos de vibración acústica, de sensibilidad del tacto y el gusto, así como la percepción de olores del olfato son transmitidos de manera constantes.

Sin embargo, Teberosky, (2000) plantea que, "Antes de comprender el modo de funcionamiento alfabético de la escritura, los niños comienzan diferenciando dibujo de escritura" (Teberosky, ob. cit., p. 3). Este proceso de diferenciación fortalece identificar las grafías de una imagen, por ende es importante que los educadores podamos propiciar en espacios de sentido crítico en la consolidación de análisis contextual, en una etapa de desarrollo neuro-perceptivo emocional de aprendizajes significativos de identificación de la estructura de las palabras en la escritura.

En efecto se resalta las consideraciones del equipo de consejería de educación de la comunidad de Madrid lo caracteriza, el "Nivel silábico" como una etapa de hipótesis silábica inicial, promueve "una correspondencia sonora (nivel silábico estricto) dando lugar a la representación de cada sílaba por la vocal" (Consejería de Educación, Comunidad de Madrid., 2003, p. 48), este nivel permite trabajar la composición de sílabas sin descuidar la fonética en el discurso de los estudiantes de primer año de escolaridad, correspondiente a estudiantes de 6 - 7 años promedio. Así mismo especifica que en el:

- Nivel silábico-alfabético, Los niños proyectan las hipótesis de cantidad, variabilidad y posición en, por lo menos, una de las sílabas.

- Nivel alfabético-medio, Proyectan la hipótesis de cantidad a otros constituyentes de la sílaba, generando la posibilidad de otras realizaciones silábicas $(\mathrm{CCV}, \mathrm{CVC}$, entre otras combinaciones (Consejería de Educación, Comunidad de Madrid., 2003, pp. 49 - 52).

Por lo tanto, el proceso de enseñanza y aprendizajes de la escritura inicia a partir del desarrollo de los niveles silábico y alfabético, que afianzan la consolidación del aprendizaje, 
en la estimulación de los procesos mentales y motrices, en la significación y percepción de contextos reales en la comprensión de la realidad, así como el desarrollo de criterios de identificación de trazos convencionales y no convencionales para la consolidación de la escritura estandarizada en los primeros años de escolaridad.

En esa perspectiva, el proceso de escritura representa una significación compleja por las dimensiones que influyen para que se pueda plasmar en un escrito, además lo qué se quiere transmitir para enseñar. Para Picardo (2005) "considera que la escritura es algo más que la transcripción de sonidos a signos gráficos" adémas recalca que, “....que la verdadera función de la escritura es comunicar un mensaje escrito" (Picardo, 2005, pp. 244 - 245), esta confirmación recobra la escencia de la escritura, estableciendo que escribir no es solamente plasmar un escrito mediante los signos o gráficos, en el fondo lleva un mensaje que implíca la intención comunicativa de aspetos influyentes que condicionan el desarrollo de la ecritura.

En ese sentido esta etapa condiciona que los estudiantes no deben solamente repetir por repetir los escritos, más bien deben expresar la comprension de sus escritos a partir de la realidad de los estudiantes, así como: las necesidades básicas de la alimentacion, el desarrollo de los hábitos de convivencia familiar y comunitaria, lo que implica la comprensión de la realidad en los procesos mentales, en la estructura de esquemas mentales, para expresar mensajes con sentido en los escritos, de esta manera se articula los contenidos curriculares de la enseñaza $y$ aprendizaje.

Al respecto Papalia, Wendkos, y Duskin (2004) expresa:
La adquisición de las habilidades de escritura está vinculada con el desarrollo de la lectura. (.....) Escribir es algo difícil para los niños pequeños. A diferencia de la conversación, que ofrece una retroalimentación constante, la escritura requiere que el niño juzgue de manera independiente si ha alcanzado su meta de comunicación (Papalia, Wendkos, y Duskin, 2004, p. 401).

A partir de este argumento se establece que las habilidades para el desarrollo de la escritura están estrechamente vinculada a la oralidad y se constituye en una necesidad para apropiarse de signos lingüísticos, tanto en el proceso de apropiación de la escritura se hace necesario que, el estudiante sienta la importancia de las metas de comunicación por medio de la escritura, en ese proceso la lectura es uno de los aspectos importantes en la adquisición de habilidades para la escritura, ya que no es lo mismo que la oralidad o el diálogo, en ese contexto existe la necesidad de trabajar las habilidades de la fonética para desarrollar la oralidad y la escucha, para contribuir de manera objetiva a la escritura, muchas veces estos elementos no son tomadas en cuenta en el proceso educativo.

En ese mismo proceso Montealegre $\mathrm{y}$ Forero (2006) establece que, "la primera fase es la preinstrumental, en la cual la escritura es un juego", (Montealegre y Forero, ob. cit., p. 27) este planteamiento expresa que el aprendizaje presenta una fase preinstrumental, relacionada a las actividades de la vida cotidiana, resaltando la importancia una fase vivencial en la apropiación en la escritura de los niños desde la enseñanza en la casa, para que las maestras de los primeros años de escolaridad puedan orientar la transición a una escritura estandarizada, dando continuidad en el desarrollo de la motricidad, en las capacidades 
lingüísticas para afianzar una escritura sólida en la enseñanza y aprendizaje.

Por consiguiente, el desarrollo de la escritura en los niños y niñas de los primeros años de la escolaridad, pasa por un proceso organizado de procesos técnico-motrices, psicomotrices en la percepción significativa y gráfica, en la praxis de la escritura. En efecto Montealegre y Forero (2006) enfatiza:

La enseñanza inicial de la lectura y la escritura ... distinguen tres etapas en el inicio escolar: a) el primer período, llamado de preparación al aprendizaje o de introducción, tiene por objeto el ejercicio de habilidades perceptivas, psicomotrices, etc., considerando que los niños tienen ya concepciones sobre el texto escrito adquiridas en contextos familiares y sociales; b) en el segundo período, se da la enseñanza sistemática de la lectura o escritura, etapa fundamental en el proceso; y c) el tercer período, el niño pasa de aprender a leer al nivel en el que lee para aprender (Montealegre $\mathrm{y}$ Forero, ob cit. p. 32).

Por lo tanto, este aporte enfatiza las etapas de preparación y estimulación en el desarrollo de las capacidades, habilidades perceptivas y motrices, en una segunda etapa considera de manera sistemática la escritura, en la tercera etapa se constituye el aprendizaje a partir de la lectura de la realidad. En tal sentido, el proceso expuesto se da de manera sistemática, simultánea con la finalidad de lograr la escritura en la diversidad de estudiantes en un determinado curso, seguidamente Montealegre y Forero (2006) establece la, "(adecuada pronunciación) y de la conciencia alfabética (reconocer que los fonemas tienen traducción gráfica); y segundo, la conciencia fonológica (reconocer componentes fónicos del lenguaje oral) y la conciencia semántica (significado de las palabras)" (Montealegre y Forero, ob. cit., p.30). Por esta razón, la escritura está relacionada estrechamente con la pronunciación, el desarrollo de fonemas condiciona la expresión adecuada y la buena ortografía en los escritos, por ello se enfatiza los procesos de comprensión de los fonemas, morfemas y lexemas en la estructura de las ideas, para desarrollar condiciones básicas de escritura

En ese sentido se comprende que, el desarrollo de la apropiación de la escritura necesariamente se organiza a partir de los conocimientos de forma estructural, abstrayendo los elementos de composición en una palabra, en ese proceso se presenta la significación fonética y morfológica, para comprender el sentido gramatical de las silabas bilíteras, monosílabas, silabas trabadas, trilíteras, consonantes y vocales, que se armonizan al momento de representar las ideas, superan la estructuración de proceso de escritura cada vez más complejas.

En esa perspectiva los procesos perceptivos facilitan en el aprendizaje concreto simbolizan la realidad de los acontecimientos vivenciales, en la codificación y la significación de cantidades, así como la comprensión del significado de los signos gráficos para la escritura.

En los procesos perceptivos se extrae información de las formas de las letras y de las palabras, y aunque las dificultades en esta fase son escasas, su evaluación se puede realizar con ejercicios como por ejemplo: buscar el elemento igual a uno dado $(\mathrm{p} / \mathrm{p} \mathrm{b}$ $\mathrm{d}$ q), o rodear el grupo silábico igual a uno dado (bar / bra bor dra bar) (Montealegre y Forero, 2006, p. 33).

Considerando la percepción de la autora, los proceso perceptivos se desarrollan en la concepción de las letras, silabas y palabras expresas en la realidad circundante y por tanto, 
no está solamente en la escolaridad, más bien se genera en diferentes actividades, que influyen en el propósito académico, tanto en el desarrollo de los planes de gestión curricular y en los planes de clases para la enseñanza y aprendizaje.

De cualquier manera, los procesos perceptivos se desarrolla a través de la lectura de la realidad, además se convierte en una estrategia para desarrollar procesos educativos dinámicos de aprendizaje, tal es el caso de la dinámica de la percepción de los sentidos sensoriales, se convierte en un canal de comunicación a la memoria activa de organización y ordenamiento de conocimientos. $\mathrm{Al}$ respecto Montealegre (2006) establece:

... con el objetivo de establecer en qué medida el conocimiento del nombre de la letra antes del inicio escolar facilita el aprendizaje de la lectoescritura, señala tres componentes importantes en la adquisición de este aprendizaje: primero, el surgimiento del procesamiento fonológico de la escritura; segundo, el aprendizaje de la correspondencia fonemagrafema; y tercero, el desarrollo de habilidades de sensibilidad fonética (Montealegre y Forero, 2006, p. 30).

En ese entendido la autora resalta el componente fonológico en el logro de la lectura y escritura y no así directamente haciendo el salto directo a las grafías o los morfemas, los fonemas presentan una fase de preparación de los sonidos para promover la apropiación efectiva de los signos lingüísticos. Por lo que la escritura pasa por una serie de etapas, en lo fonológico y morfológico en la construcción de procesos mentales en el fortalecimiento de las habilidades para el desarrollo de la escritura.

Por otra parte, el morfema es la que determina la escritura, por tanto, es un componente de la estructura de las palabras en la apropiación de los grafemas, dando sentido a los trazos de los signos lingüísticos de diferentes nomenclaturas de los signos gráficos. Al respecto Montealegre y Forero (2006) señala que, "La siguiente fase de escritura mnemotécnica indiferenciada (sin sentido), corresponde a trazos diversos con significado subjetivo para el niño", (Montealegre y Forero, ob. cit., p. 27). Este proceso asume la estimulación de la experimentación en el desarrollo de la escritura, como una etapa progresivo donde los estudiantes asumen el significado de su escrito, afianzan el ritmo procesual en la enseñanza y aprendizaje, fortalecen en la práctica de relajación y respiración que permita asumir la vocalización y la significación en la expresión verbal de la lectura de la realidad.

En efecto para el PEA de la escritura, se consideran diferentes aspectos de la realidad contextual, así sean históricos, culturales, políticos y científicos, estos aspectos representan manifestaciones innatas de la realidad, por lo tanto recobra mayor significado el desarrollo de la escritura, cuando se fortalece mediante la interacción sociocultural, "La escritura es una construcción cultural y, por tanto, adquirida socialmente muy útil para elaborar diferentes interpretaciones de la realidad personal, social, cultural, política, científica..., y para comunicar a los demás las propias fantasías e ilusiones, angustias, sentimientos, emociones..." (Ramos, 2009, p. 55), orientada en la apropiación de la escritura, con mayor significado, fomentada mediante sucesos reales en la estimulación e instrumentación para la escritura estandarizada.

En ese contexto la apropiación de la escritura se enriquece de manera apropiada en la percepción de la realidad cultural y social, con la finalidad de generar la lectura de la realidad de los aspectos comunes en nuestra vida 
cotidiana, aunque muchas veces se asume de manera equivocada, descuidando lo evidente de los aspectos intrapersonales en la producción escrita, negando el aprendizaje vivencial y emotivo que fortalece en el desarrollo de la escritura.

Así como la escritura es social, la oralidad es plenamente social al ser un canal de comunicación con sentido y contenido cultural, por lo tanto, en el proceso la escritura se enriquece de manera constante de los aspectos sociales, políticos, económicos, culturales de la realidad latente, para dar sentido al desarrollo de la escritura en nuestros estudiantes.

Así como Ramos (2009) manifiesta que, "La mayoría de las producciones escritas tienen una finalidad comunicativa, se escribe para comunicar algo a otros; sin embargo, en la escuela, la lengua escrita ha perdido su dimensión comunicativa y cognitiva (transformadora del conocimiento)" (Ramos, ob cit. p. 57). En ese sentido la escritura se hace significativa en el desarrollo de la comunicación y por la dimensión creativa de los textos escritos, lo que implica dar sentido a la escritura a partir de la lectura de la realidad concreta, rescatando las múltiples representaciones del contexto.

En esta etapa los estudiantes dan un salto cualitativo en el desarrollo de la escritura. Para Ferreiro y Teberosky en su obra, Los Sistemas de Escritura en el Desarrollo del Niño plantea la Hipótesis Silábica, "esta hipótesis el niño da un salto cualitativo con respecto a los niveles precedentes" (Medina, Fuenmayor, y Camacho, 2009 , p. 80), en ese contexto el estudiante ya tiene idea de expresar las ideas plasmadas en las silabas para desarrollar progresivamente las ideas con mayor sentido.

Sin duda las consideraciones planteadas recalca que, la enseñanza y aprendizaje de la escritura es pedagógico ante todo, es por eso que, "reconocemos que los procesos de enseñanza y de aprendizaje se dan dentro de un sistema didáctico enmarcado en contextos institucionales, y aún político sociales, que los condicionan" (Carlino, 2009, p. 22). En ese sentido se consolida la condición dialéctica, y holística de la práctica educativa y se constituye el nivel de rendimiento de las capacidades en el desarrollo de la valoración de los conocimientos, producto de la apropiación de las aptitudes en los estudiantes, como resultado del proceso de enseñanza y aprendizaje.

En ese proceso se fomenta el desarrollo de los sentidos del cuerpo, se cualifica la organización de las prioridades mentales, en una generación donde el desarrollo de las funciones sensoriales es múltiple, por la atención dispersa de los niños que han nacido en la realidad virtual del mundo cibernético. Para Álvarez (2012) "El sistema de sentidos que para el sujeto tiene el objeto, forma parte del conocimiento del sujeto, como consecuencia de la connotación que le da la persona al objeto." (Álvarez, ob. cit., p. 118) en ese sentido los procesos mentales y la realidad tangible asentuan los vinculos de asimilación en el proceso de enseñanza y aprendizaje.

Por consiguiente, el desarrollo de las competencias perceptivas de la lectura de la realidad, fortalece las capacidades de comprensión, significación y la percepción de la realidad. Por ende la percepción sensorial, promueve el desarrollo de los órganos sensoriales, lo que permite explorar la realidad del contexto que nos rodea, es uno de los aspectos elementales para la escritura, por tanto la percepción de los sentidos se constituye en un componente potencial que promueve la comprensión del mundo natural, en la lectura y en la percepción de los aspectos reales, así como el "Tálamo: estructura del tamaño de una avellana. Registra los estímulos que captan los sentidos desde el exterior) canaliza la información para que sea procesada." 
(Carminati, 2012, p. 48). En la estructura mental cognoscente de los medios que canalizan la comprensión del mundo y que se concreta en el desarrollo de la escritura convencional, dando mayor sentido al contacto con la realidad del entorno del estudiante. En ese proceso, la lectura de la realidad se establece en un proceso dinámico en el estímulo de aprendizajes, sea contrastada o anulada de acuerdo a los registros de la experiencia de cada personalidad y se establece como el instrumento esencial en la articulación de aprendizajes a partir de la lectura de la realidad.

A su vez el desarrollo de la lectura de la realidad promueve una motivación permanente en las sensaciones y percepciones del entorno que le rodea, "la motivación es una propiedad de la atención que permite seleccionar la información relevante." (Carminati, 2012, p. 81) en ese proceso se contituye el mayor interés por la asimilación y concentración para iniciar el aprendizaje y el desarrollo mental y cognitivo de pensamientos, ideas, conocimientos de la realidad vivencial. Por su parte, Woodhead y Oates (2012) establece que, "la fuerza o la debilidad de la arquitectura del cerebro, lo que a su vez determina en qué medida el niño o la niña será capaz de pensar y controlar sus emociones." (Woodhead y Oates, ob. cit., p. 4). Estos procesos mentales afianza un mejor aprendizaje en ese momento se estimula para asumir las sensaciones de acuerdo a la necesidad y motivación de cada estudiante.

Así mismo, Arrivillaga (2013) señala "la lectura oral como un conjunto de respuestas verbales articulatorias, emitidas selectivamente ante un conjunto de estímulos visuales constituidos por lo que se llama letras, sílabas, palabras o textos" (Arrivillaga, ob. cit., p. 7). Se constituye en un conjunto de respuestas verbales, evidenciada a través de los estímulos sensoriales para comprender la realidad. En ese proceso se contribuye, el desarrollo de las habilidades perceptivas a partir de la lectura de la realidad. Por lo tanto, para Álvarez (2014):

La realidad es todo lo que tiene existencia verdadera, en el espacio y en el tiempo, aquello que se refiere a las cosas. La cosa es todo lo que tiene entidad, ya sea material o espiritual. La realidad está conformada por tres tipos: la naturaleza, la sociedad y el pensamiento (Álvarez, 2014, p. 121).

En ese contexto la lectura de la realidad implica promover la connotación de las cosas y objetos con existencia verdadera en el espacio y el tiempo, para propiciar espacios de motivación permanente en el desarrollo de la escritura, aprovechando los mecanismos del organismo humano en la expresión compleja en el proceso de asimilación de los órganos sensoriales, manteniendo activo los mecanismos de servicio de información al sistema nervioso. Según Campos (2014) los "Circuitos sensoriales (vista, tacto, oído, olfato, gusto) llevan la información al sistema nervioso" (Campos, ob cit. p. 15), para promover el contacto directo con la realidad, por medio del estímulo externo en el desarrollo espontaneo de cualquiera de los órganos sensoriales, mediante la percepción de datos conocidos o nuevos en el registro de la información, en diferentes etapas de desarrollo de la lengua oral y escrita.

En ese sentido, el proceso de enseñanza y aprendizaje de la escritura se hace importante en los primeros años de escolaridad en el Modelo Educativo Sociocomunitario Productivo, por lo tanto el Ministerio de Educación (2014) plantea en los planes y programas de estudios que, "En los tres primeros años de escolaridad, el currículo relacionado con comunicación y lenguajes 
enfatiza el desarrollo de capacidades de lectura y escritura con la producción de textos gradualmente complejos, aplicando la lengua originaria, castellana y extranjera" (Ministerio de Educación 2014, p. 7). Por esta situacion, en el primer año de escolaridad se enfatiza en el proceso de enseñanza y aprendizaje de la escritura. Esto implica la enseñanza y aprendizaje de la escritura determinando la apropiación de la fonética, de acuerdo a los sonidos del alfabeto en la construcción de los morfemas, estrechamente relacionados a la estructura de las palabras, así como los grafemas que simboliza los signos de las letras en la construcción de las palabras.

Con la finalidad de promover el desarrollo de la escritura y la formación integral de los estudiantes, "En el modelo educativo el enfoque es integral, vivencial y dialógico, el cual promueve desarrollar la lengua oral y escrita a través de las habilidades lingüísticas de escuchar, hablar, leer y escribir" (Ministerio de Educación, 2017, p. 85), de esa manera se resalta la formación integral según el enfoque propuesta en el MESCP, fortaleciendo las habilidades lingüísticas, además de las habilidades neuro-perceptivas y las motrices que contribuyen en la apropiación de la escritura, fomentando el rendimiento de la escritura, como objetivo del año de escolaridad, prevista en el proceso educativo.

En el proceso educativo el rendimiento académico se constituye en un elemento importante para conocer el desarrollo integral de los estudiantes, a través de los resultados observables y evaluables en el desarrollo de las acciones que manifiestan los resultados de los aprendizajes en la adquisición de experiencias, habilidades y capacidades determinando el rendimiento de los estudiantes en el proceso educativo, en ese proceso se manifiesta capacidades mentales y físicas en el desarrollo de la escritura según el desempeño de conocimientos adquiridos en el proceso de enseñanza y aprendizaje.

Por las condiciones que profundiza las causas del problema de estudio, se define el bajo rendimiento con el aporte de la consejería de educación de la Universidad de Málaga que, el "Bajo Rendimiento Escolar es un término general que se refiere a un grupo de alteraciones en los procesos de enseñanza y aprendizaje caracterizados porque los alumnos rinden significativamente por debajo de sus capacidades" (Romero, 2004, pp. 27-28), por consiguiente, en el desarrollo de actividades, el bajo rendimiento es determinada por la alteración de capacidades que se encuentran por debajo de las expectativas en los conocimientos, capacidades, destrezas y habilidades, de carácter cognitivo, control emocional, desempeño físico, en el desarrollo de actividades prácticas que son producto de la asimilación y aprendizaje en procesos educativos.

Por consiguiente, para Castillo, (2010) "el rendimiento satisfactorio/insatisfactorio se refiere al potencial aptitudinal e intelectual del alumno desde una valoración psicopedagógica" (Castillo, ob. cit., p. 343). Esto significa que, al valorar el rendimiento se realiza una apreciación satisfactoria o insatisfactoria de los resultados que nos remite el estudiante, según el aprovechamiento como producto de los aprendizajes, en las diferentes actividades curriculares, al promover la apropiación de la escritura, mediante la significación práctica de los signos lingüísticos, de la representación gráfica de las palabras, para constatar los resultados esperados en el desenvolvimiento de las capacidades en la escritura, plasmada en mensajes concretos, en escritos o simplemente insatisfactorio en la apropiación de la escritura. 
Así mismo el rendimiento de la escritura se acompleja según los años de escolaridad, cuando el estudiante inicia la escolaridad tiene pocos desafíos, según suben los años de escolaridad existe mayores desafíos en el nivel de desarrollo de la escritura, constituyéndose en más complejo y completo el desarrollo de los componentes de un texto escrito, según Castillo (2010) plantea: "Rendimiento suficiente, y satisfactorio. Cuando el rendimiento del alumno es el que le corresponde de acuerdo a sus posibilidades". (Castillo, ob. cit., p. 342), de acuerdo al planteamiento las posibilidades de desarrollo de la escritura puede ser suficiente si corresponde a la respuestas adecuada, plasmada en ideas concretas, expresada en una ortografía adecuada, resaltada en sentido y coherencia de los mensajes escritos, por lo tanto la valoración del rendimiento en los años de escolaridad determina el rendimiento satisfactorio o el rezago en la escolaridad.

En ese sentido, para evaluar el rendimiento Castillo ob. cit., señala que, "la evaluación en los primeros cursos (evaluación globalizada): cuadernos de clase, algunos cuestionarios, escalas de observación, producciones orales, pruebas de diversos tipos, resolución de ejercicios, utilización de mapas y atlas, etc." (Castillo, 2010, p. 280). Estos avances y documentos visibles son producto de los procesos de enseñanza y aprendizaje, producto del trabajo de aula, a su vez son materiales de trabajo que se convierten motivo de valoración, para conocer los resultados en la apropiación de la escritura, determinando el nivel de aprendizaje que se alcanzó en la escritura durante el proceso de enseñanza y aprendizaje de los estudiantes.

Para dar respuesta al bajo rendimiento se adopta el análisis de la metodología, ya que representa el camino, los pasos a seguir para el logro de una meta que nos planteamos todos los días en el trabajo, en el viaje, incluso en los quehaceres de la vida cotidiana, en el desarrollo de las actividades cotidianas. Sin embargo, en el proceso educativo se convierte en una herramienta que orienta los procesos de enseñanza y aprendizaje y está estructurada en un proceso secuenciado y organizado, al respecto Álvarez (1979) plantea:
Cualquier proceso de lectura o escritura desde el punto de vista perceptivo involucra tres etapas: 1 ) la percepción global directa del estímulo o la percepción auditiva apoyada en una imagen mental: 2) el desglosamiento de la figura en cada una de sus partes; y 3) la reproducción gráfica u oral del estímulo inicial conservando sus características propias, tanto en la organización espacial como temporal (Álvarez, 1979, p. 256).

Por ello, cabe resaltar que en ese proceso la escritura es procesual en el desarrollo de las etapas, a partir de la percepción global en contacto con la realidad auditiva, visual, actividad físico motriz concreta, en la práctica de los procesos lingüísticos y orales, además de las necesidades intrínsecas fisiológicas, corporales y emotivas, se constituye el proceso de aprendizaje para el desarrollo de la escritura.

Por consiguiente, el proceso de enseñanza y aprendizaje responde a las acciones ordenadas y sistemáticas en los primeros años de escolaridad y este proceso es determinante en el desarrollo de la escritura mediante procedimientos planteados que, posibilita la lectura de la realidad en la conceptualización para contribuir al desarrollo de la escritura. Es por ello que Monereo (1999) plantea, la "sucesión ordenada de acciones que se dirigen a un fin concreto, conocido y que conduce a unos resultados precisos, respondan a una caracterización algorítmica" (Monereo, ob. cit., 
p. 10), diversificada en las actividades del proceso educativo, a fin de llevar adelante una secuencia de actividades ordenadas para el profesor y/o los estudiantes, orientado al proceso de percepción, desarrollo, aplicación, ejecución, en determinación de la enseñanza y aprendizaje y las estrategias de aprendizaje. En ese sentido, los procedimientos metodologicos promueven la organización de actividades estrategicas, orientadas al logro del objetivo del proceso de enseñanza y aprendizaje.

Al respecto Ferreiro (2005) señala que, "las relaciones sociales, ineherentes a todo proceso de aprendizaje escolar, y su insidencia en los aprendizajes curriculares" (Ferreiro, ob. cit., p. 9), estos procesos de interacción se contituye en acciones vinculantes en los procedimientos y medios para el desarrollo de conocimientos a las nuevas generaciones para expresar la realidad circundante, para permitir las prácticas sociales, y el desarrollo linguistico, al transferir y comunicar conocimientos mediante diferentes tipos de textos y en la adquisición de la escritura.

\section{CONCLUSIONES}

El desarrollo de la investigación género aportes importantes en la profundización del problema de estudio, llegando a las siguientes conclusiones:

Se determinó que, el proceso de enseñanza y aprendizaje del primer año de escolaridad en Bolivia se caracteriza por niveles de desarrollo en el rendimiento en la escritura, mediante procesos técnicos, psicológicos, biológicos, en el desarrollo de las capacidades y habilidades cognitivos y motrices para el proceso de escritura de los estudiantes.

También, se establece que, desde el punto de vista reflexivo y teórico en el proceso de enseñanza y aprendizaje de la escritura pasa por una serie de etapas, tanto en el desarrollo de capacidades y habilidades de percepción de los cinco sentidos para la lectura de la realidad, en la apropiación del signo lingüístico, en la apropiación de la psicomotricidad para la representación gráfica de las palabras, mediante los procedimientos, métodos, medios y técnicas de la metodología para el desarrollo de la escritura a partir de la realidad.

Así mismo, se considera que, el rendimiento en la escritura depende del desarrollo de percepción de los cinco sentidos, en la apropiación de las capacidades y habilidades para el proceso de enseñanza y aprendizaje de la escritura a partir de la lectura de la realidad desde lo básico hasta lo más complejo en la producción escritura.

\section{REFERENCIAS}

Álvarez, A. C. (1979). Desarrollo de las funciones básicas para el aprendizaje de la lecto-escritura segun la teoria de Piaget. Chile

Álvarez, d. Z. (2012). Epistemología del caos. Cochabamba - Bolivia: Kipus

Álvarez, d. Z. (2014). Didáctica elemental. Cochabamba - Bolivia: Grupo editorial Kipus.

Arrivillaga, 0. M. (2013). Diferencia en el rendimiento de lectoescritura en niños de primer grado primaria. Guatemala

Baqués, M. (2004). Juegos para educación infantil . España : Book print digital

Campos, A. L. (2014). Los aportes de la neurociencia a la atención y educación de la primera infancia. La Paz - Bolivia

Carlino, P. (2009). La lectura y la escritura: un asunto de todos/as. Buenos Aires

Carminati, d. L.-W. (2012). Integrando la neuroeducación al aula. Buenos Aires Argentina

Castillo, A. S. (2010). Evaluacion educativa de aprendizajes y competencias. Madrid España

Castorina, J. A. (2004). Psicología, cultura y educación perspectiva desde la obra de Vigotski

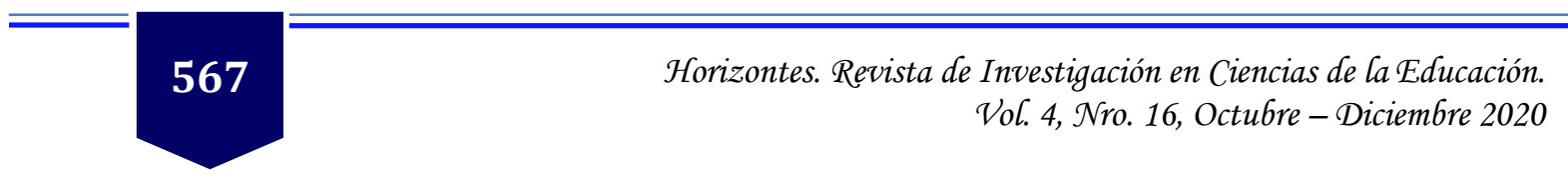


Consejería de Educación, Comunidad de Madrid. (2003). Lectura y escritura en contextos de diversidad. Madrid

Ferreiro, E. (2005). Vigencia de Jean Piaget. Mexico: Siglo XXI

Maturana, H. (1988). Extracto del texto Emociones y Lenguaje en Educación y Política. Chile

Medina, J. D., Fuenmayor, G., y Camacho, H. (2009). Psicogénesis de la Escritura: un acercamiento crítico. Red de Revistas Científicas de América Latina, el Caribe, España y Portugal, pp. 71-98

Ministerio de Educación. (2017). "El Desarrollo de la Lengua Oral y Escrita en Primaria Comunitaria Vocacional" Diplomado en Formación para el Desarrollo de la Lengua Oral y Escrita en el Modelo Educativo Sociocomunitario Productivo. La Paz, Bolivia

Ministerio de Educación. (2014). Planes y programas de estudio de Primaria Comunitaria Vocacional. La Paz, Bolivia
Monereo, C. (1999). Estrategias de enseñanza y aprendizaje. Barcelona: Graó

Montealegre, R., y Forero, L. A. (2006). Desarrollo de la lectuescritura: adquisición y dominio . Bogotá, Colombia

Papalia, D. E., Wendkos, O. S., y Duskin, F. R. (2004). Psicología del desarrollo de la infancia a la adolescencia. México

Picardo, J. O. (2005). Diccionario pedagogico. San Salvador

Ramos, J. (2009). Didáctica de la lengua / General. En Enseñar a escribir con sentido.

Rojas, B. A. (2000). "La lecto-escritura en la edad preescolar". Cuba

Teberosky, A. (2000). Los sistemas de escritura

Woodhead, M. y Oates. (2012). El cerebro en desarrollo. Reino Unido 